\title{
Phosphaturic Mesenchymal Tumor with Symptoms Related to Osteomalacia that Appeared One Year after Tumorectomy
}

\author{
Katsunobu Yoshioka ${ }^{1}$, Rie Nagata ${ }^{1}$, Mami Ueda ${ }^{1}$, Toshimasa Yamaguchi ${ }^{1}$, Yoshio Konishi ${ }^{1}$, \\ Masayuki Hosoi ${ }^{1}$, Takeshi Inoue ${ }^{2}$, Kazuhiro Yamanaka ${ }^{3}$, \\ ${\text { Yoshiyasu } \text { Iwai }^{3} \text { and Toshihiko Sato }}^{1}$
}

\begin{abstract}
A 45-year-old man was admitted to our hospital because of bone pain and hypophosphatemia. He had undergone surgery 2 years previously for a "benign unclassified mesenchymal tumor" in the skull, but there were no clinical symptoms related to osteomalacia. His laboratory examination revealed low serum phosphate, high alkaline phosphatase, and normal calcium levels. The diagnosis of tumor-induced osteomalacia due to phosphaturic mesenchymal tumor mixed connective tissue variant (PMTMCT) was made by reexamining the pathologic specimens. Oral supplementation with phosphate and 1-25-dihydroxyvitamin D relieved his clinical symptoms and laboratory values returned to normal. However, subcutaneous administration of octreotide had no clinical effect. Clinicians and pathologists should be aware of the existence of PMTMCT especially nonphosphaturic or asymptomatic variants of this disorder.
\end{abstract}

Key words: phosphaturic mesenchyal tumor, tumor-induced osteomalacia fibroblast growth factor 23 , octreotide

(DOI: 10.2169/internalmedicine.45.1797)

\section{Introduction}

Tumor-induced osteomalacia (TIO) is a rare paraneoplastic syndrome characterized by phosphaturia, hypophosphatemia and osteomalacia (1), with remission occurring after resection of the tumor. In 1987, Weidner and Santa Cruz reported that most cases of TIO are histologically distinctive and unlike any other mesenchymal tumor and coined the name "phosphaturic mesenchymal tumor mixed connective tissue variant" (PMTMCT) (2). However, most clinicians and pathologists are not aware of the existence of this type of tumor, and because PMTMCT typically has a variegated appearance, it is often misdiagnosed as another type of mesenchymal tumor.

In most cases, bone and muscle pain are the first clinical manifestations of PMTMCT, followed by identification of the tumor. Here, we report a case of PMTMCT in which symptoms related to osteomalacia appeared 1 year after tumorectomy.

\section{Case Report}

A 45-year-old man was admitted to our hospital on November 22, 2005, because of bone pain and hypophosphatemia. In June 2003, he had been admitted to the neurosurgical floor complaining of posterior neck pain and left hypoglossal nerve palsy. Clinical evidence of osteomalacia, including bone pain, was absent. Laboratory examination showed low serum phosphate $(1.9 \mathrm{mg} / \mathrm{dl})$, normal alkaline phosphatase (212 IU/l), and normal calcium $(9.1 \mathrm{mg} / \mathrm{dl})$ levels. T1-weighted magnetic resonance imaging (MRI) re-

\footnotetext{
${ }^{1}$ Department of Internal Medicine, Osaka City General Hospital, Osaka, ${ }^{2}$ Department of Pathology, Osaka City General Hospital, Osaka and ${ }^{3}$ Department of Neurosurgery, Osaka City General Hospital, Osaka

Received for publication February 28, 2006; Accepted for publication July 18, 2006

Correspondence to Dr. Katsunobu Yoshioka, Department of Internal Medicine, Osaka City General Hospital, 2-13-22 Miyakojima-Hondori, Miyakojima-ku, Osaka 534-0021
} 


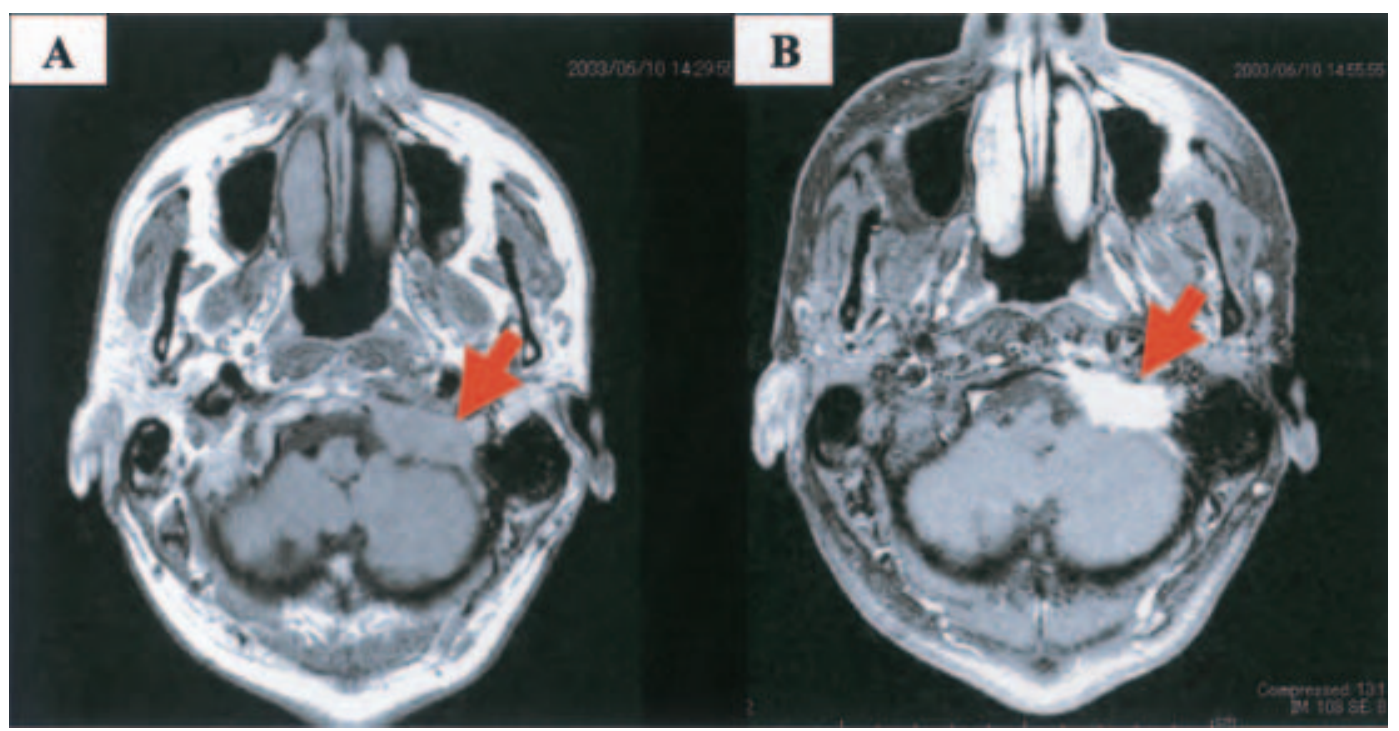

Figure 1. T1-weighted magnetic resonance imaging revealed an isointensity mass at the clivus region (panel A arrow), which was highly enhanced by gadolinium injection (panel B arrow).

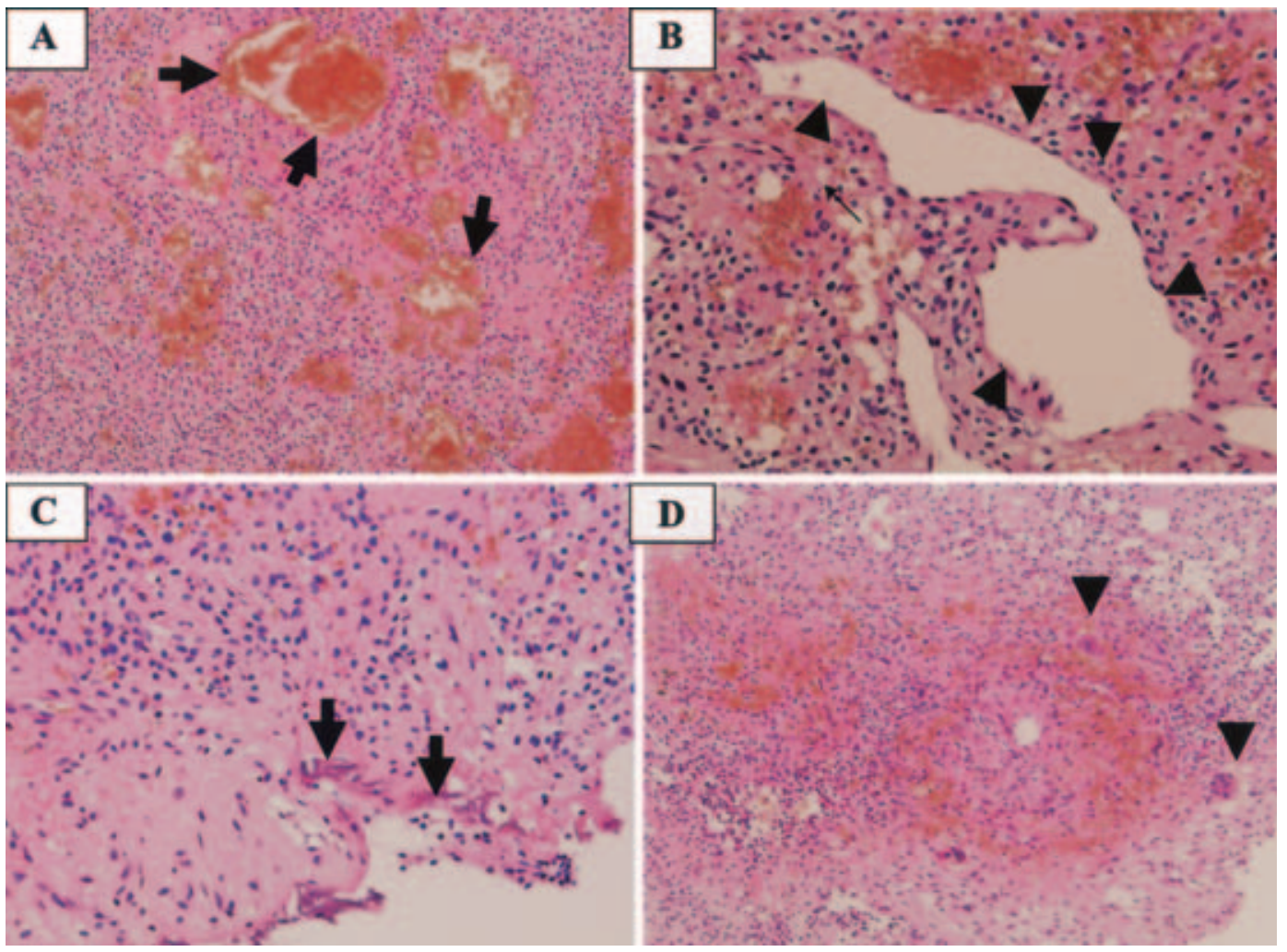

Figure 2. Histopathology of the tumor (HE stain, $\times 200)$ revealed proliferation of small round tumor cells with a well-developed capillary network (panel A arrows). Tumor cells with large nuclei were occasionally observed, but mitotic changes were not seen. Hemagiopericytoma-like vessels (panel B arrowheads), calcification (panel C arrows), osteoclast-like giant cells (panel D arrowheads), and hemorrhage were occasionally observed.

vealed an isointensity mass at the clivus region, which was highly enhanced by gadolinium injection (Fig. 1) and a subtotal tumorectomy was performed. The tumor originated in the bone and he was diagnosed with a "benign unclassified mesenchymal tumor." Unfortunately, serum phosphate levels were not measured after surgery. He became asymptomatic and was discharged. In January 2005, he became aware of pain in the ribs, which became progressively worse; the pain extended to his ankles, hip joints and other areas. Posterior neck pain recurred and MRI revealed recurrence of the tu- 


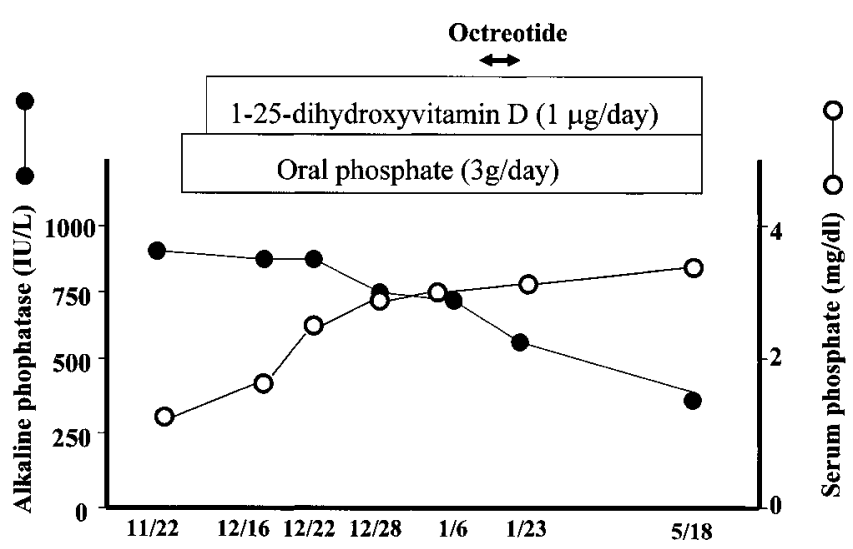

Figure 3. Serum alkaline phosphatase and phosphate levels during oral phosphate and 1-25-dihydroxyvitamin D therapy.

mor. Stereotactic gamma knife radiosurgery (12 Gy) was performed to treat the tumor. However, his condition deteriorated and whole body computed tomography revealed multiple bone fractures, including fractures of the ribs. A radionuclide bone scan revealed increased uptake in multiple bones. Metabolic bone disease was suspected, and he was referred to an internist and admitted to our department.

On admission, he was $158 \mathrm{~cm}$ and weighed $56 \mathrm{~kg}$. His temperature was $36.4^{\circ} \mathrm{C}$, pulse rate was $72 / \mathrm{min}$, and blood pressure was 122/80 $\mathrm{mmHg}$. Physical examination was unremarkable except for painful motor impairment.

The laboratory examination showed low serum phosphate (1.5 mg/dl), high alkaline phosphatase (808 IU/l), and normal calcium $(9.4 \mathrm{mg} / \mathrm{dl})$ and bicarbonate levels $(29.0 \mathrm{mEq} /$ 1). Maximum transport of phosphate in renal proximal tubules was low $(1.3 \mathrm{mg} / \mathrm{dl})$. Serum levels of parathyroid hormone were normal $(22 \mathrm{pg} / \mathrm{ml})$ and 1, 25-dihydroxyvitamin D levels were inappropriately low $(28 \mathrm{pg} / \mathrm{ml})$. There was no family history of metabolic bone disease. These findings led us to suspect TIO. Fibroblast growth factor-23 (FGF-23) level, which was measured by enzyme-linked immunosorbent assay (3), was $49 \mathrm{pg} / \mathrm{ml}$ (reference range: $10-50 \mathrm{pg} / \mathrm{ml}$ ).

We reexamined the pathological specimen to aid our diagnosis. Histopathology of the tumor revealed proliferation of small round tumor cells with a well-developed capillary network. Hemangiopericytoma-like vessels, calcification, osteoclast-like giant cells, fibrous areas, and hemorrhage were occasionally observed (Fig. 2). Tumor cells with large nuclei were occasionally observed, but mitotic changes were not seen. Results of immunohistochemistry for S-100, desmin, cytokeratin, and smooth muscle actin were all negative. These features were identical to those of PMTMCT. Thus, he was diagnosed with TIO due to PMTMCT.

The best treatment approach for TIO is to remove the tumor. However, this patient could not undergo this surgery due to tumor location; thus oral supplementation with phosphate (3 g/day) and 1-25-dihydroxyvitamin D (1 $\mu \mathrm{g} /$ day) was begun. Serum phosphate levels were gradually increased and normalized, and alkaline phosphatase levels were de-

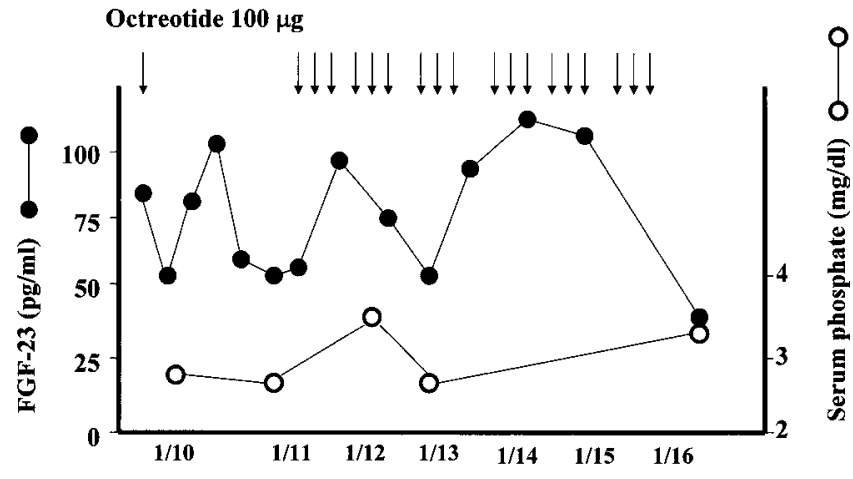

Figure 4. Serum FGF-23 and phosphate levels during subcutaneous octreotide therapy.

creased (Fig. 3), and the bone pain gradually subsided. However, the posterior neck pain became worse. After obtaining written informed consent, therapy with subcutaneous octreotide at a dose of $100 \mu \mathrm{g}$ three times a day for 7 days was started. However, it had no effect on the serum phosphate or FGF-23 levels (Fig. 4). Octreotide therapy was discontinued and he is now being treated as an outpatient receiving oral supplementation with phosphate and 1-25dihydroxyvitamin D.

\section{Discussion}

As early as 1972, Evans and Azzopardi realized that tumors that induce osteomalacia were in most cases histologically distinctive and unlike other known mesenchymal tumors (4). In 1987 Weidner and Santa Cruz termed such tumors PMTMCT (2). However, the existence of these distinct tumors is unknown by most clinicians and pathologists and many cases are diagnosed as hemangiopericytoma (5), osteosarcoma (6), and other types of the tumors. In the present case, the tumor was originally diagnosed as a "benign unclassified mesenchymal tumor."

In most cases, bone and muscle pain are the first clinical manifestations of PMTMCT, followed by identification of the tumor because the tumor is often small and localization of the tumor is difficult $(7,8)$. However, Folpe et al reported three interesting cases with histologic features identical to PMTMCT, but without clinical evidence of osteomalacia (9). They speculated that there are tumors that either secrete insufficient FGF-23 or that tumors occur in patients who are able to compensate for the increased FGF-23 secretion. Thus, PMTMCT could be nonphosphaturic in the first stage of the disease. Although hypophospatemia was present before surgery, clinical symptoms related to osteomalacia, such as bone pain, were absent in the present case. We speculate that in the second stage, PMTMCT could be asymptomatic even if phosphaturic. In the present case, 1 year after surgery, the tumor relapsed and clinical symptoms of osteomalacia appeared, suggesting that in the final stage, clinical symptoms related to osteomalacia were manifested. Clini- 
cians and pathologists should be aware of the existence of nonphosphaturic or asymptomatic variants of PMTMCT, and a patient with a tumor that is identical to PMTMCT should be carefully monitored by both imaging and laboratory studies.

TIO has recently been shown to be associated with an overexpression of FGF-23 in tumor cells, which indicates that FGF-23 is one of the circulating factors that causes renal phosphate wasting (10). It has been reported that the circulatory levels of FGF-23 are high in patients with TIO (11). However, in the present case, the FGF-23 level was not that high $(49 \mathrm{pg} / \mathrm{ml})$ compared with the levels in reported cases with confirmed TIO. Takeuchi et al reported an interesting case in whom a clinical diagnosis of TIO due to a FGF-23 producing tumor was confirmed before surgery using venous sampling for FGF-23 (7). In that case, peripheral circulating levels of FGF-23 were about 1.5-fold higher than the upper limit of the reference range $(72.9 \mathrm{pg} / \mathrm{ml})$. Thus, the circulatory levels of FGF-23 are not necessarily extremely high in patients even with a confirmed FGF-23 producing tumor. Although the mechanism of regulation of FGF-23 secretion is unknown, plasma FGF-23 levels may be regulated by serum phosphate levels because of their hypophosphate effects (12). In the present case, FGF-23 levels were considered to be inappropriately high considering his extremely low serum phosphate level. Thus, we believe that the tumor in the present case was a FGF-23 producing tumor. However, this hypothesis is not conclusive because other circulating factors such as frizzled related protein-4, or matrix extracellular phosphoglycoprotein could be the cause of renal phosphate wasting.

Recently, Seufert et al reported a man with TIO, in which the subcutaneous administration of octreotide at a dose of $50 \mu \mathrm{g}$ three times a day for 5 days and then at a dose of $100 \mu \mathrm{g}$ three times a day for 8 days abolished renal tubular phosphate wasting. They proposed that in patients in whom surgery cannot be performed, phosphate wasting may be relieved by treatment with octreotide (13). However, Paglia et al intravenously administered octreotide at a dose of $600 \mu \mathrm{g}$ per day for 6 days without any effect on the biochemical variables, just as in the present case (14). They speculated that the lack of response in their patient could be attributed to the heterogeneous distribution of somatostatin receptors among tumor cells or to the prevalence of cells lacking somatostatin receptors. Thus, it is not known yet whether octreotide therapy is effective at controlling serum phosphate levels in patients with TIO. More cases need to be compared to reach a conclusion on this issue.

In summary, we encountered a patient who was diagnosed as TIO due to PMTMCT 2 years after surgery for "benign unclassified mesenchymal tumor" in the skull. Clinicians and pathologists should be aware of the existence of PMTMCT especially nonphosphaturic or asymptomatic variants of this tumor.

This study was supported by a grant for medical research from Osaka City General Hospital. The excellent technical assistance of Ms. Nozomi Tsuji, Ms. Kanako Matsumoto, and Ms. Asami Nagata is gratefully acknowledged.

\section{References}

1. Econs MJ, Drezner MK. Tumor-induced osteomalacia--unveiling a new hormone. N Engl J Med 330: 1679-1681, 1994.

2. Weidner N, Santa Cruz D. Phosphaturic mesenchymal tumors. A polymorphous group causing osteomalacia or rickets. Cancer 59: 1442-1454, 1987.

3. Yamazaki Y, Okazaki R, Shibata M, et al. Increased circulatory level of biologically active full-length FGF-23 in patients with hypophosphatemic rickets/osteomalacia. J Clin Endocrinol Metab 87: 4957-4960, 2002.

4. Evans DJ, Azzopardi JG. Distinctive tumours of bone and soft tissue causing acquired vitamin-D-resistant osteomalacia. Lancet 1: 353-354, 1972.

5. Schultze G, Delling G, Faensen M, et al. Oncogenic hypophosphatemic osteomalacia. Dtsch Med Wochenschr 114: 1073-1078, 1989 (in German).

6. Cheng CL, Ma J, Wu PC, Mason RS, Posen S. Osteomalacia secondary to osteosarcoma. A case report. J Bone Joint Surg Am 71: 288-292, 1989.

7. Takeuchi Y, Suzuki H, Ogura S, et al. Venous sampling for fibroblast growth factor-23 confirms preoperative diagnosis of tumorinduced osteomalacia. J Clin Endocrinol Metab 89: 3979-3982, 2004.
8. Fukumoto S, Takeuchi Y, Nagano A, Fujita T. Diagnostic utility of magnetic resonance imaging skeletal survey in a patient with oncogenic osteomalacia. Bone 25: 375-377, 1999.

9. Folpe AL, Fanburg-Smith JC, Billings SD, et al. Most osteomalacia-associated mesenchymal tumors are a single histopathologic entity: an analysis of 32 cases and a comprehensive review of the literature. Am J Surg Pathol 28: 1-30, 2004.

10. Shimada T, Mizutani S, Muto T, et al. Cloning and characterization of FGF23 as a causative factor of tumor-induced osteomalacia. Proc Natl Acad Sci USA 98: 6500-6505, 2001.

11. Jonsson KB, Zahradnik R, Larsson T, et al. Fibroblast growth factor 23 in oncogenic osteomalacia and X-linked hypophosphatemia. N Engl J Med 348: 1656-1663, 2003.

12. Gupta A, Winer K, Econs MJ, Marx SJ, Collins MT. FGF-23 is elevated by chronic hyperphosphatemia. J Clin Endocrinol Metab 89: 4489-4492, 2004.

13. Seufert J, Ebert K, Muller J, et al. Octreotide therapy for tumorinduced osteomalacia. N Engl J Med 345: 1883-1888, 2001.

14. Paglia F, Dionisi S, Minisola S. Octreotide for tumor-induced osteomalacia. N Engl J Med 346: 1748-1749; author reply 17481749, 2002.

(C) 2006 The Japanese Society of Internal Medicine http://www.naika.or.jp/imindex.html 